Pedagogía y Saberes No. 50

Universidad Pedagógica Nacional

Facultad de Educación. 2019.pp. 187-198

\title{
Estructura y contenido de las prácticas preprofesionales en el modelo pedagógico de la Universidad Nacional de Educación (Ecuador)
}

Artículo de investigación

Structure and Content of Pre-professional Practices in the Pedagogical Model National of Universidad Nacional de Educación (Ecuador)

Estrutura e conteúdo das práticas pré-profissionais no modelo pedagógico da Universidade Nacional de Educação (Equador)

Lucas Palacios Liberato*

\section{Para citar este artículo:}

Palacios, L. (2019). Estructura y contenido de las prácticas preprofesionales en el modelo pedagógico de la Universidad Nacional de Educación (Ecuador). Pedagogía y Saberes, 50, 187-198.

Profesor de la Universidad Peruana Cayetano Heredia; Doctor en Educación e investigador del grupo Brain, Mind and Education.

Correo electrónico: lucas.palacios@upch.pe Código oRCID: orcid.org/0000-0002-2430-686X 


\title{
Resumen
}

Esta reflexión constituye parte de la investigación acerca de las prácticas preprofesionales de formación docente de acuerdo con el "modelo pedagógico" de la Universidad Nacional de Educación (UNAE) del Ecuador; del cual participaron los estudiantes de la carrera de Educación Básica del segundo y tercer ciclo de formación docente. El orden y el significado de las prácticas preprofesionales planteado por el modelo de la UNAE sigue los postulados científicos pedagógicos actuales acerca del desarrollo de las prácticas en la formación docente y estas también son semejantes a la experiencia de las prácticas preprofesionales desarrolladas por Walter Peñaloza en la Universidad de la Cantuta (Perú). El problema se fundamentó sobre la pregunta: ¿cómo demuestran la experiencia y los hechos que el modelo de las prácticas preprofesionales de formación docente de la UNAE se confirman como correctas? La metodología posibilitó la observación, el registro, análisis y sistematización de la experiencia, a través de la guía de observación, el diario de campo, los formatos para el diseño de un proyecto de innovación pedagógica, los otros documentos curriculares y la evaluación de los aprendizajes elaborada por los practicantes. Los resultados demuestran que los momentos de la práctica preprofesional planteados por el modelo están formulados en correspondencia con las competencias profesionales del docente y el proceso de intervención pedagógica que exige la investigación educativa.

\section{Palabras clave}

formación docente; competencias profesionales; investigación educativa; metodología de enseñanza aprendizaje

\begin{abstract}
This reflection is part of the research on pre-professional teacher training practice according to the "pedagogical model" of Universidad Nacional de Educación (UNAE) in Ecuador, with the participation of students from the BA Program in Basic Education who were in their second and third cycle of teacher training. The order and meaning of pre-professional practice proposed by the UNAE model follows the current pedagogical scientific postulates about the development of teacher training practices and that they are also similar to the experience of pre-professional practices developed by Walter Peñaloza at Universidad de la Cantuta (Perú). The problem was: How do experience and facts show that the model of pre-professional teacher training practices at UNAE is confirmed as correct? Methodology allowed the observation, record, analysis and systematization of the experience, through observation guides, field journals, the formats for the design of the pedagogical innovation project ,the other curricular documents, the learning evaluation that the students elaborated. The results show that the moments of pre-professional practice proposed by the model are formulated according to the professional skills of the teacher and the pedagogical intervention process required by educational research.
\end{abstract}

\section{Keywords}

teacher training; professional skills; educational research; teaching-learning methodology

\section{Resumo}

Esta reflexão faz parte da pesquisa sobre os estágios pré-profissionais de formação de professores segundo o "modelo pedagógico" da Universidade Nacional de Educação (UNAE) do Equador, do qual participaram os estudantes do programa de graduação em Educação Básica do segundo e terceiro ciclo de formação docente. A ordem e o significado dos estágios pré-profissionais colocado no modelo da UNAE está baseado nas abordagens científicas pedagógicas atuais sobre o desenvolvimento das práticas na formação docente e estas são, também, semelhantes às experiências dos estágios pré-profissionais desenvolvidas por Walter Peñaloza na Universidade da Cantuta (Perú). 0 problema sustenta-se na pergunta sobre: como demonstram a experiência e os fatos que o modelo dos estágios pré-profissionais de formação docente da UNAE é adequado? A metodologia possibilitou a observação, o registro, análise e sistematização da experiência, através do modelo de observação, o diário de campo, as fichas para o planejamento do projeto de inovação pedagógica, e os outros documentos curriculares, como a avaliação das aprendizagens que os estagiários fizeram. Os resultados assinalam que os momentos do estágio pré-profissional estabelecidos pelo modelo, estão formulados em relação com as competências profissionais do professor e o processo de intervenção pedagógica que exige a pesquisa educacional.

\section{Palavras-chave}

formação de professores; competências profissionais; pesquisa educacional; metodologia de ensino-aprendizagem 


\section{Introducción}

$\mathrm{E}$ 1 modelo pedagógico acerca de las prácticas preprofesionales de la Universidad Nacional de Educación (UNAE) plantea cuatro momentos en su desarrollo: comprensión-diagnóstico, diseño y planificación, desarrollo-actuación y evaluación-reformulación, esto es lo que llamamos la "estructura" que define la lógica interna de las prácticas; cada uno de esos momentos es determinado por la función y/o tarea que ella comprende, esto es el contenido. Los cuatro momentos de la práctica están organizados en correspondencia con las competencias profesionales del docente que también son cuatro fundamentales: el dominio del diagnóstico educativo, la pericia en la planificación, la capacidad de dirección del proceso de enseñanza aprendizaje y la capacidad de ejecución de la evaluación de los aprendizajes; la misma que se aplica para desarrollar la aptitud de innovación pedagógica. Todas ellas se relacionan con la investigación educativa que supone también cuatro grandes momentos: la problematización educativa, el diseño del proyecto de intervención pedagógica, la recolección de la información o los datos durante el desarrollo del proceso de enseñanza aprendizaje, y luego el trabajo de gabinete que incluye procesamiento de los datos, análisis, discusión e informe. Estas coincidencias entre las competencias, la práctica y la investigación educativa son lo que han venido trabajando y planteando Paulo Freire (Brasil) en el modelo de educación liberadora, por procesos de concientización, acción, reflexión, acción transformadora; o Elliott (2010) junto a otros, en la metodología de la lesson study, que implica diagnosticar el problema, plantear la estrategia de solución, aplicar y evaluar para reprogramar y volver a desarrollar la intervención pedagógica. Particularmente, Walter Peñaloza (Perú) lo desarrolló en la formación docente en la Universidad de la Cantuta, de donde provenimos, en el modelo de prácticas preprofesionales que se iniciaba desde el primer ciclo de la carrera profesional docente. Al respecto, Peñaloza afirma que esta,

no era un curso para ir al aula y escuchar lecciones de un profesor. Era acción real de los futuros maestros frente a los niños y a los jóvenes. Como acción y trabajo real, se ubicaba a gran distancia de las asignaturas y representaba el momento en que todo lo recibido en las asignaturas debía traerse a cuento apropiadamente para afrontar los hechos que iban surgiendo en la escuela y en el aula. (Peñaloza, 1989, p. 196).

Semejante preocupación e interés tiene el modelo UNAE, al esperar que las prácticas se articulen con las asignaturas que se dictan simultáneamente en el aula de la facultad; aunque este propósito resulta mucho más complicado no es contrario a un interés ideal en la formación docente, por cuanto la práctica sirve para poner a prueba la utilidad de las asignaturas en los hechos, como que se demuestre su relación con la acción real del futuro docente frente a los niños, ya que,

una vez visualizada la sustancia de esta práctica, que era y es trabajo y no asignatura, y que es trabajo relacionado con la profesión, resultó fácil darle una nueva ubicación (la que siempre tuvo per se, aunque no se lo viera). (Peñaloza, 1989, p. 195).

Es decir, si las asignaturas desarrollan las competencias profesionales del docente, las materias de las asignaturas deben servir para el trabajo real y concreto en las aulas con los niños. Sin embargo, el estado del arte actual acerca de las competencias profesionales del docente, demuestran que dichas competencias se desarrollan fundamentalmente en la práctica más allá de los cursos teórico dictados en las salones de clase de la facultad.

Queríamos nosotros, no una educación intelectualista y academizante sino una educación plena, en la que hubiera el contacto con los conocimientos (esfera de la verdad científica), pero, asimismo, la oportunidad de penetrar en otras manifestaciones de la cultura, mediante una serie de actividades (no asignaturas ni lecciones) en las que los alumnos fueran los protagonistas. Y anhelábamos que los educandos se comportaran no únicamente como receptores, sino que ejercieran sus capacidades creativas, tanto en lo cognoscitivo, como en lo no cognoscitivo. (Peñaloza, 1989, p. 191).

Si a ello le sumamos la necesidad de ejecutar paralelamente a la práctica una investigación acerca de la intervención pedagógica, necesitamos una concepción holística e integral de las prácticas preprofesionales en la formación docente. Esperando que las prácticas resulte la "piedra de toque" de todas ellas, que demuestren la utilidad de las asignaturas, que se constituyan en el momento para investigar, en el lugar para la intervención pedagógica o la innovación didáctica, como para el desarrollo completo de las competencias docentes. Que comprendan, ante todo, una investigación diagnóstica para la acción pedagógica, la planificación de la acción docente, la dirección del proceso de enseñanza aprendizaje y la evaluación de los aprendizajes. Como afirma Peñaloza, la práctica preprofesional del futuro docente,

tiene que ser completa, y esto exige que practique no solo en el dictado de clases (práctica docente), sino guiando actividades de los niños y jóvenes en 
la escuela o el colegio y adiestrándose en los procedimientos administrativos propios de los colegios y escuelas. (Peñaloza, 1989, p. 199).

Así, el desarrollo de las competencias profesionales del docente, la investigación, la innovación pedagógica y la aplicación teórica de las asignaturas requiere y exige la práctica de la prueba en casos y situaciones reales como único criterio de verdad; entonces, la práctica no es una preocupación casual del modelo UNAE, sino un interés común que comparte con otras experiencias educativas anteriores, y por ello su estructura y contenido se ajusta a las exigencias de diversas investigaciones, teniendo en cuenta que toda acción docente empieza por el diagnóstico, sigue con la planeación, la dirección del proceso de enseñanza aprendizaje y culmina con la evaluación de los aprendizajes. Este orden y regularidad debe seguir toda práctica profesional desde el primer momento de la formación de la carrera docente y de esta forma constituir el núcleo de las experiencias fundamentales; que, por presentar casos o hechos reales, enriquece la teoría pedagógica y la investigación educativa. Así, los futuros maestros movilizan en la práctica todas sus facultades, aptitudes, competencias, verificando la utilidad, funcionalidad o aplicabilidad de la teoría pedagógica. Por ello, Freire indicaba,

No hay enseñanza sin investigación ni investigación sin enseñanza. Esos quehaceres se encuentran cada uno en el cuerpo del otro. Mientras enseño continúo buscando, indagando. Enseño porque busco, porque indagué, porque indago y me indago. Investigo para comprobar, comprobando intervengo, interviniendo educo y me educo. Investigo para conocer lo que aún no conozco y comunicar o anunciar la novedad. (Freire, 2004, p. 14).

No es posible desarrollar las competencias profesionales del docente desligándolas de la investigación científica en general como de la investigación pedagógica en particular, la práctica preprofesional debe integrar en un solo proceso la teoría y la práctica investigativa, en la acción docente, y esta permitirá a la vez desarrollar las competencias profesionales. Como indica Julio Barreiro, resumiendo el modelo de educación freiriano, "la educación verdadera es praxis, reflexión y acción del hombre sobre el mundo para transformarlo" (Barreiro, 2004, p. 7). En otras palabras, no podemos interpretar que la formación docente sea verdadera praxis si no integra la teoría y la práctica investigativa como acción pedagógica durante las prácticas, que por ello resultan fundamentales, no accesorias, suplementarias o complementarias. El desarrollo de la investigación a través de la acción docente no solo resulta indispensable sino inevitable, por cuanto promueve la investigación y desarrolla la innovación pedagógica, como indica Perrenoud

creo que esto podría ser un método - entre otrospara llevar a cabo en cualquier investigación-acción, investigación-desarrollo o innovación referente a las prácticas: intentar explicar las competencias que faltan, a partir del análisis de las prácticas, pero también utilizando un referencial existente, orientado hacia competencias emergentes, para "releer" lo que ya se hace o lo que se pretende hacer. (Perrenoud, 2004, p. 147).

En este punto, la práctica preprofesional docente no solo sirve para aplicar la teoría de las asignaturas, sino también para desarrollar la investigación $\mathrm{y}$ ante todo las competencias profesionales, que los estudiantes de la carrera docente ingresen a las escuelas desde el primer ciclo para vivenciar in situ las condiciones reales de la profesión durante los estudios correspondientes; como indica Ángel Pérez, estas sucedan "cuando los practicantes desarrollan la experimentación en grupos, por el cual diagnostican, diseñan, eligen el tema, diseñan, planifican, desarrollan, discuten, evalúan" (Pérez, 2015, s/p.). Y, es lógico creer que para ello los estudiantes de las prácticas previamente puedan haber recibido la teoría fundamental y necesaria, pero ante todo mucho más importante que esa teoría la reciba simultáneamente a la práctica para entenderla y asumirla mejor, de lo cual deben encargarse los tutores de la práctica, por cuanto,

Para acompañar, ayudar y experimentar, necesitarán todos los contenidos todo el proceso de la formación docente y en especial de nuestras disciplinas cuyos conceptos sean útiles para comprender el aula, para diseñar una actividad, pero las prácticas no son el territorio donde se aplica lo que se pretende en la teoría. Es el espacio en donde la teoría se muestra útil para poder desarrollar esas actividades. (Pérez, 2015, s/p.).

La práctica en la formación de la carrera docente define la indagación educativa como investigaciónacción por cuanto resulta simultáneamente la prueba o el ensayo de una innovación pedagógica, cuyo estudio trata acerca de la intervención pedagógica. Este es un postulado que reclaman todas las experiencias acerca de la práctica docente, como investigación e innovación pedagógica; así lo intuye Ángel Pérez, cuando afirma que la práctica es "quien marca el sentido y la naturaleza de las prácticas es la escuela, nuestro modelo pedagógico, es acompañar, ayudar y experimentar. Vamos a acompañar, a ayudar y a 
experimentar" (Pérez, 2015, s/p.) porque la "práctica" no es solo la puesta en hechos de lo aprendido sino un proceso de aprendizaje real y necesario, de aprendizaje verdaderamente significativo durante la formación docente. La práctica somete a "prueba de fuego" el desarrollo de las competencias profesionales pensados en teoría, debe estar ligada a la investigación educativa y a la innovación pedagógica, de lo contrario:

Los que prefieren que nada cambie solo tienen que hacer referenciales que se distancien de las prácticas más sencillas de justificar en el claroscuro de la tradición del "todo el mundo lo hace". La sensatez y el acuerdo tácito sobre la esencia del oficio echan un velo protector sobre la realidad de las prácticas. El debate sobre los referenciales concierne, sin embargo, a los que actúan en el cambio hacia la profesionalización y para justificarlo, deben apoyarse en el estado actual de las prácticas. (Perrenoud, 2004, p. 149).

En resumen, un buen modelo de práctica pre profesional planteado por la academia, la universidad o la facultad debe articular, integrar, nuclear todos los elementos de la formación docente, aprovechar su desarrollo en la "práctica", como se intuye en el modelo de la UNAE, por "el hecho de que los interesados se encarguen de su propia formación continua es uno de los índices más seguros de profesionalización de un oficio" (Perrenoud, 2004, p. 151). Y, a continuación, las definiciones de cada uno de los momentos de la práctica preprofesional en el modelo pedagógico de la UNAE

- Diagnóstico/exploración, es el momento destinado a identificar los problemas y necesidades del aprendizaje del educando en la escuela, como sujetos de aprendizaje a cargo; comprende la formulación del problema, la definición de la necesidad o la deficiencia de atender singular y específicamente, a fin de desarrollar la "capacidad para comprender y diagnosticar situaciones, procesos y sistemas" (Pérez, 2015, s/p.).

- Diseño/planificación, es el momento para plantear las estrategias, métodos o proyectos de solución de los problemas y necesidades identificadas anteriormente. Comprende la definición de los propósitos y las acciones correspondientes para la solución del problema; la organización del objetivo, el contenido, el método y la evaluación como elementos del proceso de enseñanza aprendizaje; la formulación del método es la "capacidad para diseñar, desarrollar y evaluar de manera personalizada el currículum" (Pérez, 2015, s/p.).
- Desarrollo/actuación, es el momento de la realización y/o ejecución de la clase o la lección en el aula con el grupo correspondiente, la práctica en sí, la aplicación del proyecto y actuación del docente en los hechos, el ejercicio de docente del practicante. Comprende la dirección real y concreta del proceso de enseñanza-aprendizaje tal como fue planificado anteriormente, destinado a observar los cambios y/o progresos de los aprendizajes del educando, registrando los logros del aprendizaje, la modificación de la conducta, el comportamiento de los educandos, etc. Enfrentando todo aquello que la interacción docente/educando y contexto pueda generar para seguir aprendiendo. Y, entonces, desarrolla la "capacidad para diseñar y construir contextos y comunidades de aprendizaje" (Pérez, 2015, s/p.).

- Evaluación/análisis, es el momento para evaluar el proyecto, la estrategia o el método aplicado, como intervención pedagógica, sobre la base de la práctica o la acción docente. Comprende el análisis y la reflexión acerca de los cambios reales en el aprendizaje, el comportamiento del educando, la influencia del proceso y contexto educativo sobre la base del proyecto desarrollado, comparando las condiciones de entrada y de salida, respecto del problema que se ha intentado resolver, la atención de la necesidad o la deficiencia superada, destinada a desarrollar la capacidad de "aprender a autorregularse y a desarrollarse profesionalmente a lo largo de toda lo vida" (Pérez, 2015, s/p.).

Como ya indicamos, este modelo de práctica debe permitir el desarrollo simultáneo de las competencias profesionales del docente, la investigación educativa como intervención pedagógica, la prueba o ensayo de la innovación pedagógica, la aplicación de la teoría de las asignaturas para observar su utilidad o funcionalidad en relación con la formación docente, $\mathrm{y}$ ante todo verificar la posibilidad de integrar o articular todos los elementos de la formación docente.

\section{Metodología de trabajo}

Este trabajo corresponde a la labor que desarrollamos como tutores de la práctica preprofesional de treinta estudiantes de la carrera de Educación Básica, en la Escuela de Educación General Básica Emilio Abad del Cantón de Azogues, provincia del Cañar, cuando cursaban el segundo y luego el tercer ciclo, entre octubre de 2015 a julio de 2016. Para desarrollar el Proyecto Integrador de Saberes (PIS) 
y asumir la conducción de las clases o lecciones como el acompañamiento de catorce educandos, los participantes se organizaron en pares, las prácticas se desarrollaron en el turno matutino, los grados que asumieron fueron de segundo a sexto de Educación General Básica. De las clases o lecciones en el aula, para las cuales fue necesario diagnosticar, planificar y luego evaluar, los participantes desarrollaron tres clases o lecciones como mínimo durante el semestre, aplicando los momentos de la lesson study.

El método de investigación del presente trabajo se resume en tres procesos sucesivos: la observación de la acción docente y/o la práctica del participante, el registro de los hechos con todos los instrumentos de investigación aquí descritos y la sistematización cuyo resultado se publica. La lógica de esta estrategia se describe en el mismo sentido de cómo se desarrolla la actividad pedagógica, por cuanto la investigación educativa no es ajena a la lógica del fenómeno estudiado ni a la disciplina correspondiente; en otras palabras, la metodología de la investigación se define en términos pedagógicos, tal como indica el orden de los momentos de la práctica, articulando el desarrollo de las competencias profesionales, la investigación educativa y la innovación pedagógica, como sigue.

1. La primera tarea de este proceso de investigación se inicia cuando el practicante registra el problema de aprendizaje que debe atender en el aula de clase y su grupo correspondiente; con la ayuda de la guía de observación. Como parte del momento del diagnóstico o la exploración debe seleccionar, elegir y definir el problema de modo claro y preciso. Respecto del desarrollo de la competencia profesional docente esto significa realizar el diagnóstico educativo respondiendo a la pregunta: ¿cuáles son las necesidades, deficiencias, problemas fundamentales y urgentes que se deben atender respecto de los aprendizajes de los educandos? En relación con el proceso de investigación debe transformar el problema de aprendizaje en un problema de investigación sugiriendo una determinada estrategia. El momento del diagnóstico también significa el punto de origen de la innovación para la intervención pedagógica, ya que el problema asumido será resuelto a través de una estrategia para mejorar los aprendizajes, orientados a corregir la deficiencia con un plan de acción pedagógica.

2. Definido el "problema", lo que sigue es el diseño o la construcción de la estrategia didáctica como parte del momento de diseño/pla- nificación. El objetivo es proponer y elaborar un modelo de prueba para experimentar en las clases como parte de la investigación, la estrategia didáctica que será ensayada en el grupo de educandos a cargo. Particularmente, respecto al desarrollo de la competencia profesional docente, este momento significa el desarrollo de la pericia en la planificación curricular por la cual se organizan los objetivos, contenidos, métodos y la evaluación del proceso de enseñanza-aprendizaje en un documento o diseño de proyecto. Respecto de la innovación pedagógica, la propuesta didáctica permitirá realizar la intervención pedagógica sobre el grupo de educandos. En este preciso momento el problema pedagógico se convierte en un problema de investigación porque permite relacionar el factor de deficiencia de aprendizaje (problema pedagógico) con la estrategia de solución didáctica, obteniendo una variable independiente (la estrategia didáctica) y la variable dependiente (la deficiencia de aprendizaje a superar) Aquí un ejemplo ilustrativo de diferencia entre el problema pedagógico y el problema de investigación, tal como fueron planteados por los practicantes.

- Problema pedagógico: deficiencias de los educandos al leer textos escritos o impresos.

- Problema de investigación: ¿Cómo desarrollan la capacidad de leer textos escritos o impresos las estrategias lúdicas?

3. Definidas las estrategias didácticas, los practicantes desarrollan las clases o sesiones previstas según su planificación; dicha actividad es registrada en el diario de campo, describiendo el momento de desarrollo/actuación; para la práctica docente significa el momento fundamental: la lección o clase en sí. Para el desarrollo de la competencia profesional docente esta corresponde al desarrollo de la capacidad de dirección del proceso de enseñanza aprendizaje en el aula de clase con el grupo educando a cargo. El diario de campo se convierte en un instrumento vital y neurálgico de toda la práctica, de la misma forma que para la investigación, porque ayuda a registrar los datos e informaciones, base sustancial de la investigación empírica, acerca del proceso de intervención pedagógica; la innovación pedagógica en este caso se convierte en el objeto de estudio de la investigación, del practicante y de la pedagogía. La clase se convierte 
en el centro de interés o eje de correlación de la investigación, para el desarrollo de las competencias y la innovación pedagógica; la lección permite registrar la acción docente durante la experimentación y/o desarrollo de la estrategia didáctica diseñada.

4. Después de todos los procesos anteriores, deviene el momento de la evaluación/análisis, que puede resumirse así: primero, la evaluación de los aprendizajes que desarrolla el practicante para observar cómo la estrategia que aplicó, mejoró o ayudó a superar las deficiencias iniciales de los educandos. Segundo, la evaluación acerca del desarrollo de cada una de las clases o lecciones, principalmente entre el tutor de aula y el tutor académico de la universidad con los participantes de la práctica. Tercero, la evaluación general de la práctica que se desarrolla en la universidad entre los tutores académicos y los practicantes acerca de todas las tareas relativas a práctica en sí, el desarrollo de las competencias docentes, la investigación y la innovación pedagógica durante la práctica. En el caso de la UNAE, el instrumento o documento que resumía todo el proceso de la práctica, la investigación y la innovación, durante la experiencia fue el Proyecto Integrador de Saberes (PIS), luego remplazado por otro documento y proceso, desterrando el enfoque científico de la práctica porque supuestamente era dañino para la formación docente. Otros instrumentos que se usaron para presentar la experiencia investigativa y pedagógica de la práctica fueron el artículo científico y las diapositivas acerca de las estrategias didácticas diseñadas y aplicadas por el practicante, todas presentadas al término de las prácticas; estas permitieron evidenciar el grado y nivel de desarrollo de la investigación educativa, la experimentación de la estrategia didáctica y el desarrollo de las competencias profesionales del docente. Todo ello en correspondencia con el momento de evaluación/análisis según el modelo UNAE.

Esto condujo al proceso de sistematización de la experiencia, que se inició desde el primer momento de la práctica, en cuanto registra, sistematiza e informa lo observado y experimentado en cada momento de las prácticas. Respecto de nuestra experiencia en la escuela de educación básica, la estrategia didáctica aplicada por los practicantes sobre el problema pedagógico permitió indicar los cambios o mejoras en el aprendizaje que generó la estrategia en los educandos, haciendo que los practicantes pudieran manifestar sus comentarios, reflexiones o conclusiones al terminar sus prácticas. En el caso particular del semestre abril-julio de 2016, las prácticas fueron desarrolladas de forma intensiva en solo dos semanas con una asistencia diaria a la escuela, las actividades se aplicaron a modo de lesson study o de reflexión-acción-reflexión como indica Freire. Para el tratamiento de los resultados del aprendizaje, acerca de cada estrategia didáctica, los practicantes presentaron la estadística correspondiente a la evolución de los aprendizajes de los educandos como consecuencia de la aplicación de la estrategia didáctica propuesta frente a los problemas educativos que le dieron origen.

\section{Materiales e instrumentos}

Los instrumentos de investigación que permitieron sistematizar nuestra experiencia, analizar las prácticas docentes como objeto de estudio y cumplir con los requisitos de la investigación, articulando el desarrollo de las competencias profesionales, la investigación educativa y la innovación pedagógica, durante el desarrollo de los cuatro momentos de las prácticas preprofesionales según el modelo UNAE, fueron los siguientes:

a. Guía de observación del problema. Instrumento diseñado para que los practicantes puedan identificar las dificultades, necesidades o deficiencias en el proceso del aprendizaje de los educandos en la escuela; su formato detalla las referencias que deben tenerse en cuenta en la observación, la exploración o el diagnóstico educativo, y concluye con la formulación del problema pedagógico. Sugiere un conjunto de preguntas a considerar acerca del entorno escolar, el clima general de la escuela, el ambiente del aula de clase. Plantea la observación de la clase o lección del docente de la escuela a cargo, la organización de la clase para las actividades, la planificación, el desarrollo de la clase o lección, el dominio de contenidos por parte del docente, las actividades del aula, el aprendizaje de los alumnos, la evaluación de las actividades; sugiere observar y/o revisar los cuadernos de trabajo de los alumnos. Esta guía constituye las primeras tareas de los practicantes, en el momento inicial de la práctica, desarrollado con el apoyo del tutor de la universidad; la guía es entregada por los practicantes el primer día de las prácticas, sobre cuya base se realizaron las prácticas siguientes en la escuela. 
b. El diario de campo. Instrumento diseñado para realizar una observación sistemática de la práctica, por lo que los practicantes lo presentan cada vez que visitan la escuela y hacen sus tareas; allí registran por escrito lo observado, la reflexión y análisis correspondiente. El formato contiene los datos informativos: escuela, lugar, nivel o modalidad, paralelo y/o grado al que asiste el practicante, nombre del practicante, fecha de prácticas, número de la práctica, tutores de la universidad y docente de la escuela. El cuerpo o contenido del diario se divide en tres componentes; el primero dedicado al desarrollo/actuación que precisa la palabra clave o descriptor; la actividad realizada u observada, aquella actividad desarrollada por el docente y/o la actividad desarrollada por el practicante, define en lo fundamental la estrategia empleada en clase. La segunda parte también exige la palabra clave o descriptor acerca de la exploración/ diagnóstico, orientado a formular el problema o la incidencia observada en clase, debe precisar la situación o el evento acontecido durante la clase, la fecha y hora del registro, los actores involucrados. La tercera parte comprende el apartado de la evaluación, para formular la reflexión, el juicio crítico o las conclusiones acerca de las estrategias o actividad docente observadas, o acerca de su propia "práctica". Dicho instrumento es presentado y revisado por el tutor después de cada día de prácticas.

c. Formato del diseño del proyecto didáctico. Instrumento que constituye un apoyo o guía para la formulación de la estrategia didáctica que debe plantear el practicante antes de las clases o lecciones a su cargo. En él organiza los propósitos de la estrategia, indica el contenido de aprendizaje, la metodología a desarrollar y la forma de evaluar dicha clase o lección, distribuida en tres cuerpos o tres tablas de doble entrada. La primera tabla comprende tres columnas; en la primera columna se formula el problema pedagógico o el problema de aprendizaje; en la segunda se define el concepto involucrado en el problema que permita sugerir una estrategia didáctica, y en la tercera se indica la solución pedagógica consecuente con el problema indicado. El segundo cuadro, también dividido en tres columnas, corresponde a la definición del proyecto innovador; la primera columna destinada a formular la idea del proyecto o el título de la estrategia; la segunda columna para la definición de la estrategia, para describir el concepto de la estrategia; la tercera columna para indicar el objetivo de aprendizaje que desarrolla la estrategia didáctica, precisando los resultados a observar y/o verificar. El tercer apartado corresponde a la descripción de la metodología, en lo fundamental se exige ordenar la secuencia lógica de las tareas de la estrategia didáctica correspondiente a la clase o lección, por la cual se enumeran en estricto orden las acciones, procedimientos u operaciones de la clase o lección; a ello se agregan tarea/funciones del docente practicante durante el desarrollo de la estrategia. La tercera columna indica el cronograma de aplicación de la intervención pedagógica, la experimentación de la estrategia didáctica, señalando de modo aproximativo el tiempo en minutos dedicados a cada apartado de la clase o lección. Debajo de todo ello se pide indicar los recursos o materiales.

d. Documento y/o productos de la experiencia. Desde el inicio de la práctica se exigió la presentación del PIS como informe de investigación o sistematización de la práctica, a ello se agregó el artículo científico para la evaluación por pares de su experiencia desarrollada y la sustentación pública por los participantes en las instalaciones de la universidad; nuestros grupos de tutoría a cargo, además prepararon las diapositivas acerca de la estrategia didáctica. Los instrumentos y formatos adicionales los planteamos con el apoyo de la coordinación de prácticas, pero posteriormente fueron prohibidos por las autoridades universitarias. En realidad no se trata de tres tareas y productos distintos u opuestos sino de productos que se complementan en el Proyecto Integrador de Saberes (PIS), de ella se resume el artículo científico y las diapositivas solo reproducen lo estrictamente didáctico, la innovación que provocó la intervención pedagógica en la escuela con los educandos a su cargo.

- El formato del PIS exigía precisar la problemática, el marco teórico, la metodología, los resultados y la discusión. La evaluación del PIS tenía en cuenta la correlación que debía existir entre el problema pedagógico que dio origen a la estrategia didáctica y el problema de investigación, la metodología como desarrollo de la estrategia didáctica, el momento experimental o la práctica en sí, según el modelo UNAE. Los resultados de investigación presentaban los logros de aprendizaje de los educandos en calificaciones, índice de promedios obtenidos 
por el grupo de educandos, comparaciones entre la evaluación de la prueba de entrada y de salida.

- El formato del artículo científico debía reproducir a modo de síntesis (1) el resumen o abstract de la investigación; (2) el problema y el objetivo de la investigación; (3) la metodología, los medios y materiales; (4) los resultados, como logros de aprendizaje de los educandos, diseñados en figuras o gráficos estadísticos, y (5) la discusión para formular las conclusiones o recomendaciones.

- La estructura de las diapositivas que se presentó en la escuela en la que se desarrollaron las prácticas precisaba en lo fundamental la estrategia didáctica aplicada, la descripción de la "secuencia lógica de tareas" y los resultados de la evaluación de los educandos o los logros de los aprendizajes como producto de la innovación aplicada.

\section{Resultados}

De los documentos y/o instrumentos presentados por los practicantes que permitieron sistematizar la práctica planteada por el modelo UNAE, enumeramos lo observado y evaluado, respecto del desarrollo, logros y las peculiaridades que describen la presente experiencia; estas, en particular, nos indican cómo se relaciona la práctica con el desarrollo de las capacidades de investigación, de innovación pedagógica y el desarrollo de las competencias profesionales del docente, como detallamos a continuación.

\section{Del diagnóstico/exploración}

a. En el semestre de octubre de 2015 a febrero de 2016 se identificaron los siguientes problemas pedagógicos, como parte del momento de diagnóstico/exploración: "problemas en la destreza escritura, en cuanto confunden, omiten y/o aumentan letras al escribir", "dificultades en la lectura-escritura, en el momento de copiar las tareas que la docente pone en la pizarra, en el momento de realizar ejercicios en el libro y en el momento de resolver las pruebas", "dificultades en la comprensión de las lecturas, cuentos y conceptos presentados en los textos educativos", "dificultades de escucha y expresión de los pensamientos propios y los de sus compañeros, en el momento de la clase como en el momento del receso", "bajo nivel de comprensión lectora"; que "no existe compañerismo en el aula", "poca o nula relación de amistad o compañerismo dentro del salón de clase, distanciamiento entre los estudiantes y frecuencia de bromas o burlas", falta de un "clima educativo apropiado en el aula de clases a causa de la indisciplina", "falta de motivación de los niños y desperdicio de sus habilidades motrices", los niños son "hiperactivos, movidos, inquietos, lo que les impide aprender". A partir de estos problemas pedagógicos se formularon las siguientes preguntas de investigación del PIS: “ ¿cómo desarrollar las potencialidades de los niños para contribuir a su desenvolvimiento cognitivo?", “¿por qué los niños no escriben ni leen correctamente?", “¿cómo lograr que el estudiante aplique correctamente las reglas ortográficas?", “ipor qué los niños y las niñas no pueden formular el enunciado que tiene un problema y a la vez resolverlo?", etc.

b. La problemática del momento de diagnóstico/ exploración del semestre de abril a julio de 2016 fueron: "dificultades en la fluidez de la lectura y la comprensión del texto", "dificultades en la comprensión lectora", "deficiencia en la comprensión lectora, en distintos niveles, al tratar los textos en clase", "falencias en el proceso de lectoescritura, tales como faltas de ortografía y la comprensión de textos", "los niños no saben describir, ni analizar fenómenos aunque los vean, no diferencian las características internas de las externas", "confusión en el uso de las consonantes, como por ejemplo, no escriben la primera letra de sus nombres y apellidos con mayúsculas, no escriben con mayúsculas los nombres propios y de lugares", "dificultades en el dominio de las reglas ortográficas, en especial con el uso de la 'll', 'y', 'b' y 'v',', “dificultades en la comprensión de lecturas, cuentos y conceptos presentados en los textos educativos", "dificultades en el momento de producir nuevos textos o reproducir textos partiendo de una lectura dada", "deficiencia de la comprensión lectora al realizar tareas relacionadas con la lectura", "dificultades en la redacción de textos descriptivos, evidenciado en el desarrollo de estudios sociales cuando redactan textos".

\section{Del diseño/planificación}

a. Los diseños de la estrategia didáctica que formularon los practicantes en el semestre de octubre de 2015 a febrero de 2016 fueron: “El 
espantapájaros", "Dominó de letras", "Pintar palabras", "La guía turística", "El anillado", "Láminas de secuencia", "Descubre la palabra", "Resolución del problema matemático en una ilustración gráfica”, "Imagen-letra”, "Sopa de letras", "Léemelo". "Juego de vedoques", “Sabia, la guacamaya", "Lectura colaborativa”.

b. Los diseños didácticos correspondientes al semestre de abril a julio de 2016 fueron: "lectura compartida de cuentos cortos", "flash cards", "lluvia de ideas", "comprensión del texto y redacción de pequeños textos", "resumen sobre lo aprendido", "uso de la ' $m$ ' antes de " $p$ " y "b" y el uso de la " $h$ ", "palabras y oraciones con las letras ' $\mathrm{ll}^{\prime}$, ' $\mathrm{y}$ ', 'b' y 'v'”, "recrear los textos de manera apropiada", "diseño del comic infantil", "crean un nuevo cuento", "reflexionar e interpretar la lectura", "creación de textos descriptivo".

\section{Del desarrollo/actuación}

a. Las clases o lecciones que observamos durante el semestre de octubre de 2015 a febrero de 2016 fueron las actividades de lectoescritura, dinámicas grupales e individuales, actividades lúdicas o técnicas basadas en el juego, taller de lecturas y producción del texto escrito, organización, ensayo y presentación de teatro, desarrollo del festival deportivo.

b. Las clases o lecciones correspondientes al semestre de abril a julio de 2016 replicaron estrategias didácticas del semestre anterior, modificadas o corregidas, aplicando un control riguroso del desarrollo de la estrategia didáctica para observar la mejora de los aprendizajes de los educandos; por ello este desarrollo o actuación de la práctica, como experimento pedagógico, fue seguido a modo de lesson study, aplicando en todos los grupos una prueba de entrada y de salida acerca de los aprendizajes de los educandos, el mismo que fue procesado como producto o resultado de la estrategia desarrollada.

\section{De la evaluación/análisis}

a. La evaluación de los aprendizajes, el control de las mejoras que produjo el experimento, los resultados que obtuvo cada estrategia innovadora aplicada por los practicantes se observan en la siguiente figura. En ella se describe la evolución de a prueba de entrada con la evolución de la prueba de salida por los distintos grupos que la desarrollaron.

\section{Evolución de los aprendizajes}

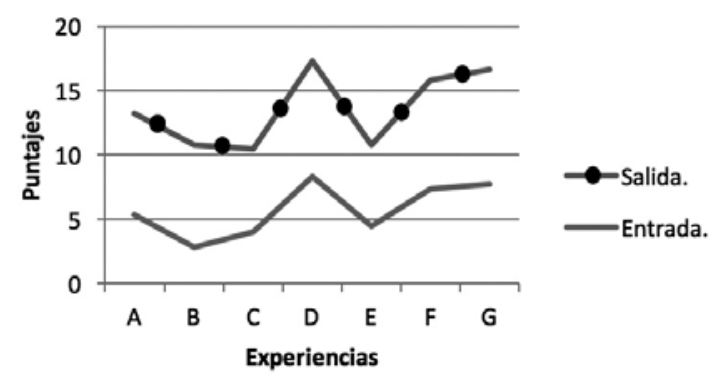

Figura 1. Evolución de los aprendizajes de los educandos bajo la conducción de nuestros practicantes

Fuente: resumen de las evaluaciones que los practicantes desarrollaron e informaron. De elaboración propia.

b. Del análisis que agregan los participantes a modo de juicio crítico o a modo de conclusión respecto de su experiencia, estas fueron sus formulaciones: la "intervención a través de las técnicas de aprendizaje basadas en el juego" incrementó los promedios de las clases; "los test de entrada y salida", permiten "darnos cuenta que en la lectura los niños demuestran tener un mejoramiento"; "podemos darnos cuenta que el uso de la estrategia lectura-escritura cooperativa ha sido de gran utilidad para mejorar las habilidades en la lectura-escritura"; "pudimos evidenciar que los estudiantes tenían mucho interés en realizar las entrevistas, pues se mostraron deseosos por aplicar la técnica"; la "metodología aplicada demostró que la problemática del aula debía ser tratada con muchas más intervenciones para poder evidenciar resultados más significativos"; luego de la práctica "se requiere más de una intervención para evidenciar mejores resultados"; "hemos dedicado a la práctica ha sido de mucha calidad e interacción con la docente y estudiantes"; hemos logrado "un acercamiento positivo hacia la comprensión de la lectura" en cuanto "los alumnos demostraron el gusto e interés" a través "de la música y el teatro"; "las actividades lúdicas, permite que los niños desarrollen la interrelación con el medio"; se crearon "un espacio de sociabilización, en el que puedan aprender y mejorar el aprendizaje", donde los niños "pueden manifestar alegría, emoción y sobre todo cooperación y trabajo en equipo"; se "logró reforzar la automotivación y el trabajo en grupo", en cuanto 
los "estudiantes se mostraron activos en el aprendizaje, se pudo reforzar lo aprendido", se observó "el cómo realizaron el resumen de los aspectos centrales del tema del video y en la construcción del cuento"; los niños "en el transcurso de las actividades planificadas de la obra teatral, mostraban mayor interés y participación involucrándose con los personajes representados", logrando "integración y participación de niños rezagados" en la "elaboración de cuentos grupales"; se desarrollaron los aspectos "espacial, interpersonal e intrapersonal", como una mejora "en el desempeño al realizar los trabajos encomendados", "acrecentar la confianza en sí mismos, al presentar sus trabajos al grupo"; los niños llegaron a "comprender cómo el lenguaje escrito representa las ideas y los conceptos" respecto de los objetos en el mundo real y el lenguaje oral en su cultura, "permitiéndoles codificar las letras y formar palabras y oraciones empleándolas correctamente en cada oración"; las estrategia "ayudó a los niños a mantenerles ocupados, así los niños se sentían motivados para trabajar", también contribuyó con el aprendizaje de los niños "encaminados a un fin específico"; "la conversación y la observación de los gráficos, aprendieron a producir un enunciado correctamente, ya que de esta forma se les facilita identificar los datos y/o cantidades".

\section{Discusión}

En primer lugar, la experiencia demuestra que la propuesta de los cuatro momentos de la práctica planteados por el modelo UNAE, no solo por responder a las exigencias de las investigaciones pedagógicas actuales y el desarrollo de otras experiencias, como la peruana de la Cantuta y Peñaloza, son correctas por cuanto así lo demuestran los hechos. La investigación verifica que en la práctica preprofesional se articulan las competencias, la investigación y la innovación; se integran las diferentes tareas docentes en cada uno de sus momentos; los instrumentos usados permiten aportar información para observar los momentos de la práctica: las competencias del docente, la investigación educativa y la innovación pedagógica; así tenemos en un solo proceso la investigación educativa, el desarrollo de las competencias profesionales, el proceso de investigación educativa como sistematización de las prácticas, la innovación pedagógica desarrollados en cada uno de los momentos de la "práctica" planteada en el modelo UNAE.

Tabla 1. Correlaciona el orden de los momentos de la práctica,

las competencias, la investigación y la innovación

\begin{tabular}{|c|c|c|c|}
\hline Momentos de la práctica & Competencias docentes & Investigación educativa & Innovación pedagógica \\
\hline Diagnóstico/exploración & Diagnóstico educativo & \multirow[b]{2}{*}{ Problematización } & Identificación del problema \\
\hline Diseño/planificación & Planificación & & $\begin{array}{l}\text { Diseño de la pro- } \\
\text { puesta innovadora }\end{array}$ \\
\hline \multirow[b]{2}{*}{ Desarrollo/ actuación } & \multirow{2}{*}{$\begin{array}{l}\text { Dirección de proceso de } \\
\text { enseñanza-aprendizaje }\end{array}$} & Experimentación & Ejecución de la innovación \\
\hline & & $\begin{array}{c}\text { Recojo o registro de } \\
\text { datos e informaciones }\end{array}$ & $\begin{array}{l}\text { Observación y regis- } \\
\text { tro de la experiencia }\end{array}$ \\
\hline \multirow{3}{*}{ Evaluación/análisis } & \multirow{3}{*}{$\begin{array}{l}\text { Evaluación de los } \\
\text { aprendizajes }\end{array}$} & $\begin{array}{l}\text { Procesamiento de los } \\
\text { datos e informaciones }\end{array}$ & \multirow[t]{2}{*}{ Análisis de los resultados } \\
\hline & & Análisis de los resultados & \\
\hline & & Discusión e informe & Informe \\
\hline
\end{tabular}

Fuente: elaboración propia.

En ese sentido la "práctica" resuelve las preocupaciones teóricas de Perrenoud respecto al desarrollo de las competencias docentes; se adecua al proceso de la lesson study de Elliot como método de investigación. La propuesta de Ángel Pérez en la UNAE es equivalente a la experiencia de la Cantuta de Walter Peñaloza acerca de cómo organizar y desarrollar las prácticas preprofesionales; también resuelve la preocupación de Paulo Freire acerca de la necesidad de hacer la investigación como un proceso de acción y reflexión permanente.

Si bien es cierto que el modelo UNAE no plantea literalmente, en principio, la relación de la práctica preprofesional con la investigación científica, esta 
exige un PIS como consecuencia natural de ella. El modelo UNAE está preocupado por el desarrollo de las competencias profesionales del docente desde el inicio de la formación docente o la carrera profesional, le preocupa la necesidad de innovar la práctica pedagógica como contribución a la solución de la problemática escolar ecuatoriana. Es una pena que las autoridades de la UNAE hayan postergado el rigor lógico científico de las "prácticas" y hayan prohibido el desarrollo de la investigación científica, ignorando su enlace natural con la experiencia in situ, la necesidad del control o seguimiento de la práctica. Por lo demás, queda demostrado que los momentos de exploración/diagnóstico, de diseño/planificación, de desarrollo/actuación y evaluación/análisis organizan la estructura y el contenido de las prácticas preprofesionales de la carrera docente.

Respecto de la relación de las "prácticas" con la teoría de las asignaturas, se exige un trabajo singular para verificar su aplicación de ella; es necesario demostrar la aplicación de la teoría de las asignaturas en la práctica para verificar su utilidad en la carrera docente como su pertinencia en el plan de estudios; creemos en especial que las "asignaturas" de investigación científica e innovación pedagógica deben desarrollarse a través de las "prácticas preprofesionales"; no es posible que se reduzcan al simple dictado de la teoría en las aulas de clase de la universidad; del mismo modo, la innovación pedagógica como desarrollo del proceso de enseñanza aprendizaje solo puede concebirse en la práctica para superar el estigma de la transmisión de los conocimientos en las aulas de clase de la facultad. Así, todos estos procesos no pueden suceder por separado, desarti- culados o atomizados, como defecto teórico contrario a la realidad objetiva, las prácticas preprofesionales constituyen el nexo, centro o eje de articulación observados en la presente experiencia.

\section{Referencias}

Barreiro, J. (2004). Educación y concienciación. En Freire, P. (2004). La educación como práctica de libertad. México: Siglo XXI.

Elliott, J. (2010). El "estudio de la enseñanza y del aprendizaje": una forma globalizadora de investigación del profesorado. Revista Interuniversitaria de Formación del Profesorado, 68 (24, 223-242).

Freire, P. (2004a). Pedagogía de la autonomía. São Paulo: Paz y Tierra.

Freire, P. (2004b). La educación como práctica de la libertad. São Paulo: Paz y Tierra.

Peñaloza, W. (1989). La Cantuta. Una experiencia en educación. Lima: Conytec.

Pérez, A. (2015) Conversación desarrolladas en la UNAE, el 21 de octubre de 2015. Transcripción.

Pérez, A y Soto, E. (s.f.). Las Lesson Study. ¿Qué son? Recuperado de http://www.ces.gob.ec/doc/8tavoTaller/ metodologa\%20lesson\%20study.pdf

Pérez, A. (s. f.). Nuevos perfiles. Las competencias profesionales de los docentes. Taller 3, de la Formación y Perfeccionamiento de los coordinadores curriculares de las carreras de educación. CEs Ecuador.

Perrenoud, P. (2004). Diez nuevas competencias para enseñar. Barcelona: Graó. 